\title{
Visualization of hepatic arteries with 3D ultrasound during intra- arterial therapies
}

\author{
Maxime Gérard ${ }^{1}$, An Tang ${ }^{2,3}$, Anaïs Badoual ${ }^{4}$, François Michaud ${ }^{3}$, Alexandre Bigot ${ }^{3}$, \\ Gilles Soulez ${ }^{2,3}$, Samuel Kadoury ${ }^{1,5}$ \\ ${ }^{1}$ Institute of Biomedical Engineering, Polytechnique Montréal, 2900 Edouard-Monpetit Bld, \\ Montreal, QC, H3T1J4, Canada \\ ${ }^{2}$ Department of Radiology, Radio-Oncology and Nuclear Medicine, Université de Montréal, \\ Montreal, QC, H3C 3J7,Canada \\ ${ }^{3}$ Centre de recherche du Centre hospitalier de l'Université de Montréal (CRCHUM), Montreal, 900 \\ Saint-Denis St, QC, H2X 0A9,Canada \\ ${ }^{4}$ Institute of Microtechnology, Ecole Polytechnique fédérale de Lausanne, Lausanne, CH-1015, \\ Switzerland \\ ${ }^{5}$ Department of Computer Engineering, Polytechnique Montréal, 2900 Edouard-Monpetit Bld, \\ Montreal, QC, H3T1J4, Canada
}

\begin{abstract}
Liver cancer represents the second most common cause of cancer-related mortality worldwide. The prognosis is poor with an overall mortality of $95 \%$. Moreover, most hepatic tumors are unresectable due to their advanced stage at discovery or poor underlying liver function. Tumor embolization by intra-arterial approaches is the current standard of care for advanced cases of hepatocellular carcinoma. These therapies rely on the fact that the blood supply of primary hepatic tumors is predominantly arterial. Feedback on blood flow velocities in the hepatic arteries is crucial to ensure maximal treatment efficacy on the targeted masses. Based on these velocities, the intra-arterial injection rate is modulated for optimal infusion of the chemotherapeutic drugs into the tumorous tissue. While Doppler ultrasound is a welldocumented technique for the assessment of blood flow, 3D visualization of vascular anatomy with ultrasound remains challenging. In this paper we present an image-guidance pipeline that enables the localization of the hepatic arterial branches within a 3D ultrasound image of the liver. A diagnostic Magnetic resonance angiography (MRA) is first processed to automatically segment the hepatic arteries. A non-rigid registration method is then applied on the portal phase of the MRA volume with a 3D ultrasound to enable the visualization of the 3D mesh of the hepatic arteries in the Doppler images. To evaluate the performance of the proposed workflow, we present initial results from porcine models and patient images.
\end{abstract}

Keywords: hepatic arteries segmentation, intra-arterial procedures, 3D visualization, ultrasound, MRI, multi-modality registration

\section{INTRODUCTION}

Intra-arterial therapies for primary liver cancer rely on the fact that the blood supply of these tumors is predominantly of arterial origin ${ }^{1}$. There are several variants of intra-arterial techniques, including intra-arterial hepatic chemotherapy which consists of the infusion of chemotherapeutics drugs in the hepatic artery through a catheter. The tip of the catheter may be positioned at the gastroduodenal artery - hepatic artery junction or further in intra-hepatic branches ${ }^{2}$. In trans-arterial chemoembolization, anticancer drugs, often mixed with Lipiodol (oil-based contrast medium), are injected into the hepatic artery. Embolizing agents are then administered to the patient to occlude the tumor-feeding arteries. This induces stasis in the targeted vessels, prevents wash-out of the drug and provokes tumor necrosis ${ }^{3}$. Drug eluting beads transarterial chemoembolization is a recent technique in which a hydrogel is injected that continuously releases the

Medical Imaging 2016: Image-Guided Procedures, Robotic Interventions, and Modeling,

edited by Robert J. Webster III, Ziv R. Yaniv, Proc. of SPIE Vol. 9786, 97862

(C) 2016 SPIE · CCC code: $1605-7422 / 16 / \$ 18 \cdot$ doi: $10.1117 / 12.2217450$ 
chemotherapeutic agent while occluding the tumor vascularization. This new drug delivery system reportedly enhances tumor exposure while reducing systemic exposure ${ }^{4}$.

In such therapies, blood flow velocities along the planned path are necessary to modulate the injection rate to achieve an optimal effect on the tumors. While Doppler ultrasound is a well-accepted and safe technique for blood flow assessment, the visualization of hepatic arterial branches with ultrasound remains challenging and is further complicated by a high frequency of anatomical variants. Fusion of diagnostic magnetic resonance angiography (MRA), where the hepatic arteries are visible after contrast administration, with intra-procedural ultrasound may be used to precisely localize these arteries during the procedure.

Fiducial-based registration techniques rely on external markers, bony landmarks or manually given markers to perform the alignment, while image-based techniques use organ surfaces or vascular structures as features to guide the registration process. For intraoperative use, manual interactions should be kept to a minimum. Few automatic ultrasound-magnetic resonance imaging (MRI) registration methods have been described in the literature. Lange et al. ${ }^{5}$ developed a fast nonrigid registration method based on vessel centerlines as a technique for mapping preoperative models into intraoperative 3D ultrasound. Their registration process made use of a combination of the Iterative Closest Point (ICP) algorithm and multilevel B-Splines and required minimal user interaction (a first step consisting of coarse rigid alignment of the vessels centerlines required 3 to 4 manually given paired landmarks). Porter et al. ${ }^{6}$ presented a two-step rigid approach that used correlation between vessels meshes segmented on both modalities to register the 3D MRI and the 3D ultrasound. Fuerst et al. ${ }^{7}$ described an intensity-based registration scheme that adapts the $\mathrm{LC}^{2}$ metric, which the authors originally developed for CT-ultrasound registration to the case of MRI-intraoperative ultrasound registration for neurosurgery. The $\mathrm{LC}^{2}$ metric compares the ultrasound intensities to a locally variable linear combination of MRI intensities and gradient.

The objective of this work is to assess the feasibility of integrating a segmented 3D mesh model of the liver hepatic arteries obtained from diagnostic MRA into 3D ultrasound acquired during live intra-arterial interventions. This would allow rapid visualization the hepatic arteries in the US volume and facilitate acquisition of US-Doppler measurements on those arteries for administration of embolizing agents based on specific patient anatomy.

\section{METHOD}

The complete workflow for hepatic arteries visualization in 3D ultrasound is shown in Figure 1. The inputs of the pipeline include a preoperative portal phase MRA, an arterial phase MRA, and an intra-procedural 3D ultrasound of the liver. The output displays the 3D mesh model of the hepatic arteries within the ultrasound volume, which can be reformatted in different planes.

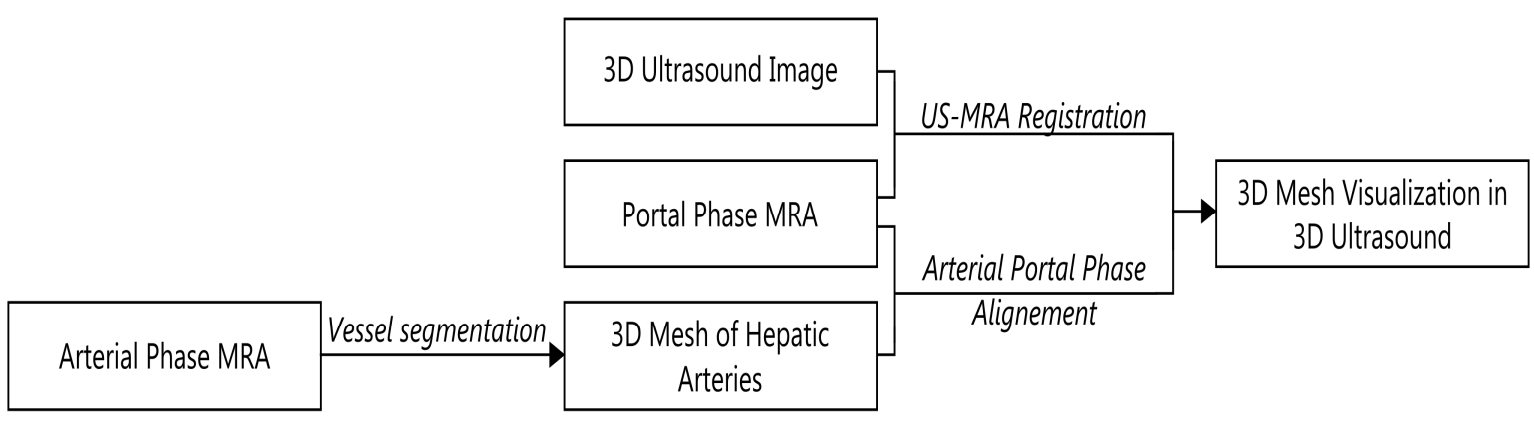

Figure 1: Workflow for visualization of hepatic arteries in 3D ultrasound.

\subsection{Image acquisition}

Prior to therapeutic intervention, contrast-enhanced MRA images were acquired after injection of a gadolinium-based contrast agent. For the porcine models, thoe images were acquired on a 3T Skyra MR system (Siemens Medical 
Solutions, Erlangen, Germany) using a T1-weighted 3D gradient-recalled echo (GRE) sequence. The MRA images of the human patient were acquired on a 3T Achieva system (Philips, Amsterdam, The Netherlands) using a T1-weighted 3D fast field echo (FFE) sequence. Arterial phase images were acquired 15-20 seconds after contrast agent injection and portal phase images 65-70 seconds after injection.

All ultrasound images were acquired on the EPIQ G7 system (Philips, Amsterdam, The Netherlands) using an X6-1 abdominal matrix probe. 3D images were acquired with a wide sweep angle and adequate depth to cover as much liver as possible. Ultrasound images were acquired at the beginning of the procedure, prior to chemoembolization.

The images of the 7 porcine models were acquired with respect to the guidelines of the Canadian Council on Animal Care and the Research Ethics Board of Health Canada and the Public Health Agency of Canada.

\subsection{Vessel segmentation from MRA}

The 3D mesh model of the hepatic arteries is automatically extracted from 3D arterial-phase MRA using an adaptation of the spinal cord segmentation tool developed by De Leener et al. ${ }^{8}$.

As described by Badoual et al. ${ }^{9}$, this segmentation algorithm starts by automatically detecting the aorta on axial slices of the MRA using an adaptive Hough transform. The junction between aorta and celiac trunk is then detected on the image using intensity and proximity constraints as well as spatial clustering to distinguish the celiac trunk from the mesenteric artery. The orientation of the celiac trunk is subsequently determined using a local contrast analysis. From there, a multihypothesis tracking module segments the centerlines of the arterial tree, and tags each of the tree's bifurcations. Finally, the vessel walls are segmented by propagating tubular deformable mesh models along the detected centerlines. The propagation process consists of the iterative duplication of a small segment of the tubular mesh. This segment is then oriented according to the previously segmented centerline and then deformed to match the vessel walls.

\subsection{Ultrasound-MRA registration}

Ultrasound-MRA registration was performed using the portal-phase images because portal veins are larger, easier to visualize and can be seen further at the periphery of the liver whereas hepatic arteries are only seen well centrally using ultrasound. Furthermore, hepatic arteries are known to follow the ramifications of the hepatic portal system. Thus, portal veins seemed to be a valid common feature to be used for the registration of both MRA and 3D ultrasound.

As a consequence of using portal-phase MRA for registration, arterial and portal phases of the MRA had to be aligned prior to MRA-US registration. This allows for the use of the transformation computed on portal-phase images to map the 3D arterial mesh into the 3D ultrasound image. The arterial-portal phase co-registration was conducted using an affine transformation in order to correct for slight motion discrepancies between both acquisitions.

Once both phases are aligned, registration is performed between the 3D ultrasound and the portal phase MRA images using a combination of landmark and image-based registration approaches. An approximate rigid approach co-aligns the volumes based on corresponding landmarks, followed by a non-rigid transformation that is applied to the US volume in order to map the US image to the portal phase MRA. The multimodal image registration can be achieved using a highorder Markov Random Field (MRF) optimization method ${ }^{10}$ using a local cross-correlation metric adapted for ultrasound and MRI imaging. Qualitative results for multimodal images from both porcine and human models are presented in the results section. Initial quantitative results are also presented for a set of 7 porcine models.

\section{RESULTS}

\subsection{D segmentation of hepatic arteries}

First, the early-phase MRA is processed to automatically segment the hepatic arteries. Figure 2a depicts the 3D mesh of the splenic and hepatic arteries of a porcine model as generated by the segmentation algorithm applied on the arterial phase MRA. The common hepatic artery, the proper hepatic artery, the left branch of the hepatic artery and a gastric 
artery are visible on the mesh. Figure $\mathbf{2 b}$ shows the result of the segmentation algorithm on a patient MRA with liver cancer. Both left and right branches of the hepatic artery are visible, as well as the gastro-duodenal artery defining the separation between the common and proper hepatic arteries. When comparing the results yielded by the algorithm to those by manual segmentation, the mean square distances (MSD) between the 3D surface meshes was of $0.69 \pm 0.28 \mathrm{~mm}$ and of $1.49 \pm 0.51 \mathrm{~mm}$ between the $3 \mathrm{D}$ centerlines.

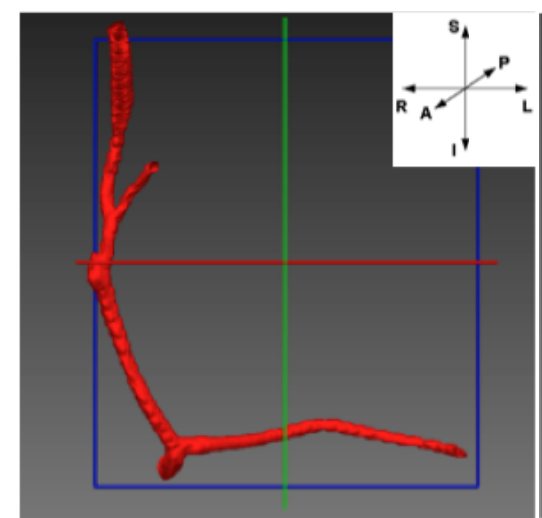

(a)

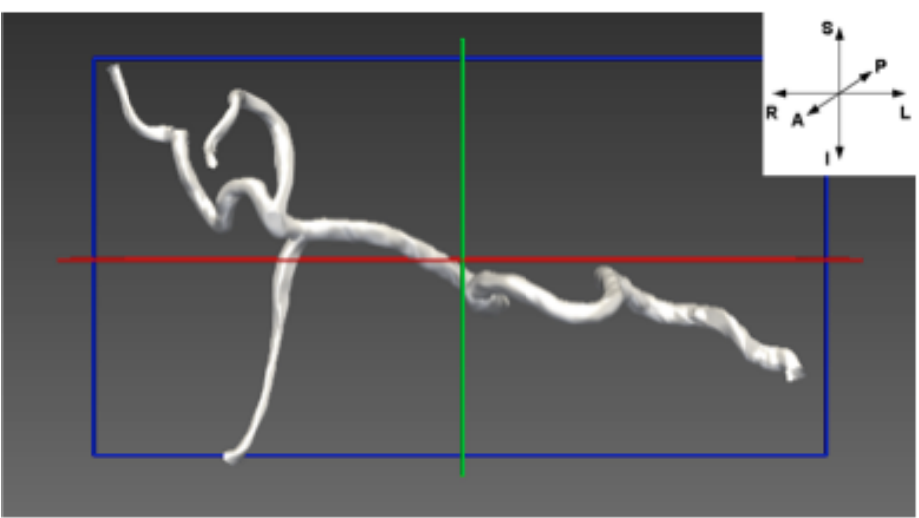

(b)

Figure 2 - (a) 3D hepatic artery segmentation in (a) a pig and (b) in a human patient.

\subsection{Ultrasound-MRA registration}

As previously discussed, an alignment between arterial and portal phases must be performed before the MRA-ultrasound registration is conducted. Results of the alignment process are depicted below. Figure 3a shows the overlay of the arterial mesh (white) segmented from the arterial phase onto the volumetric reconstruction of the portal phase MRA (red). Figure 3b represents a coronal slice of the two superposed volumes where the portal phase is in purple and the arterial phase in green.

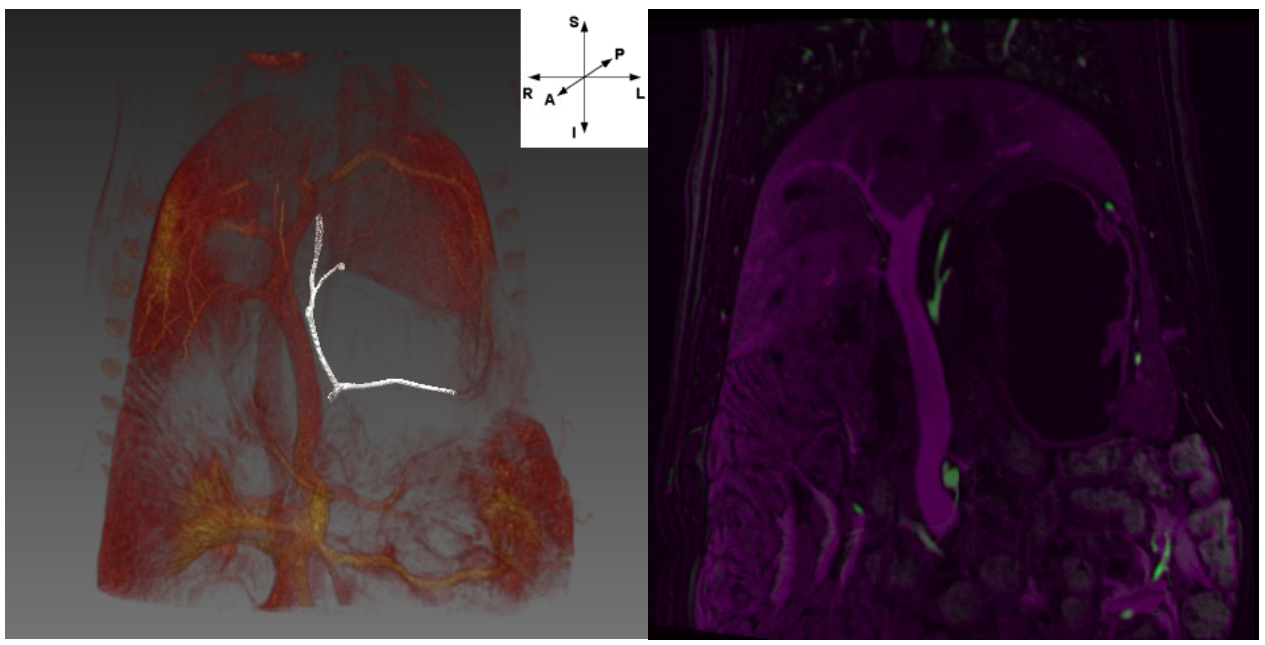

(a)

(b)

Figure 3 - (a) 3D mesh segmented on arterial phase (white) depicted within portal phase volume reconstruction (red).

(b) Coronal slice: arterial (green) portal (purple) phases aligned.

Portal-Phase MRA images were then used to perform the registration between the MRA and 3D ultrasound spaces. Our routine was successfully tested on 7 porcine models and a human patient. 
Figure 4 shows the result of the ultrasound-MRA registration for a porcine model. The displacement of the liver's boundaries between MRA and registered ultrasound may be due to mild deformation induced by breathing.

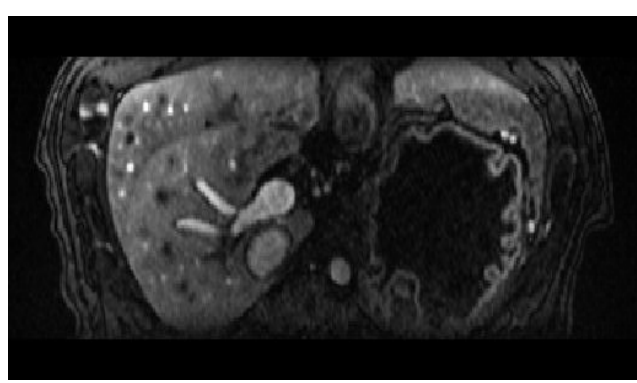

(a)

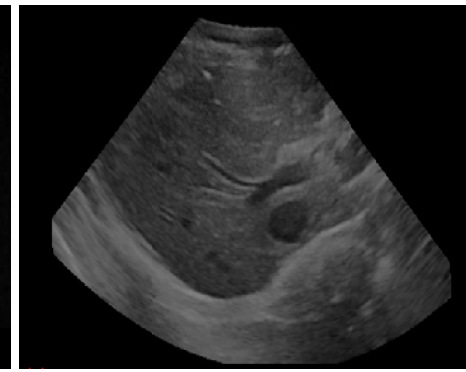

(b)

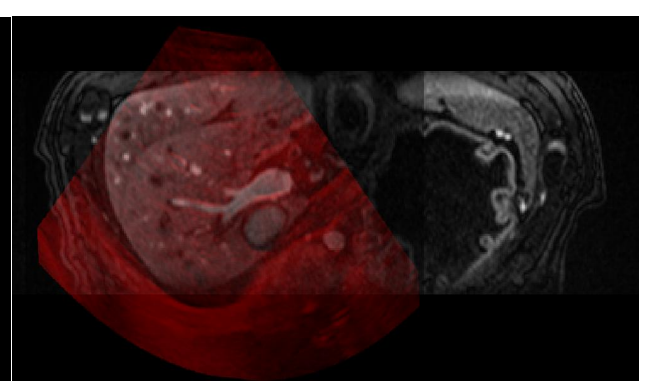

(c)

Figure 4 - (a) Portal-phase MRA of the pig's liver. (b) Corresponding ultrasound image of pig's liver. (c) Overlay of ultrasound-MRA registration.

Figure 5 shows the result of the non-rigid registration of the patient's images. Accurate matching of both vessels and liver's edges is achieved.

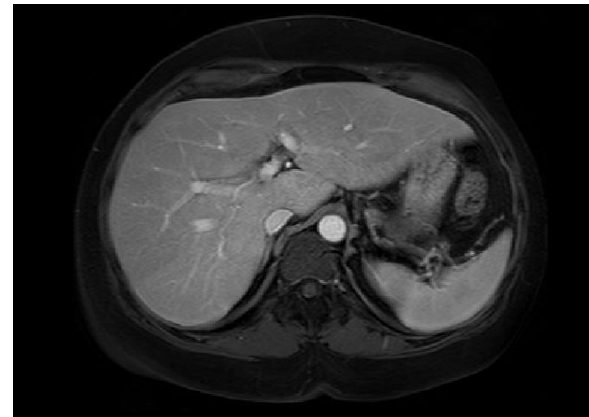

(a)

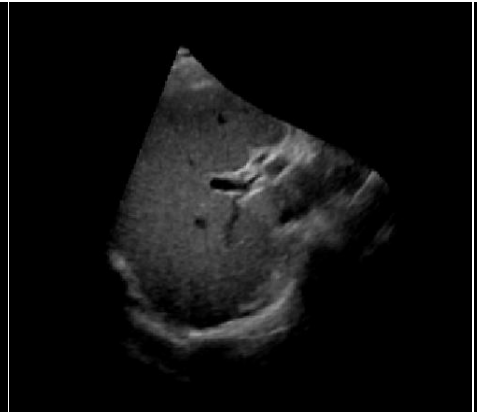

(b)

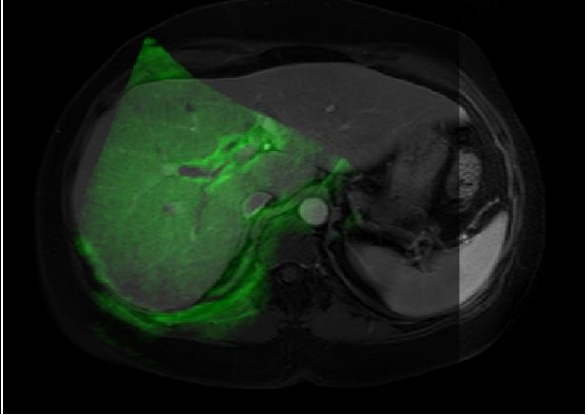

(c)

Figure 5 - (a) axial slice of MRA on a patient. (b) Result of US-MRA registration. (c) Registered 3D ultrasound of human liver.

Multi-modal registrations were evaluated in terms of target registration error (TRE) on 7 porcine models. For each pair of ultrasound-MRA images, 4 portal vein bifurcations, identified in both modalities, were manually selected as target points. The root mean square distances (RMS) between the MRI targets and the corresponding registered ultrasound target points were then measured to evaluate the error for each alignment. The registration results for the 7 porcine models are listed in Table 1.

Table 1. Target Registration Error (TRE) for US-MRI registration on 7 pigs

\begin{tabular}{|l|c|c|c|c|c|c|c|}
\hline & Pig 1 & Pig 2 & Pig3 & Pig 4 & Pig 5 & Pig 6 & Pig 7 \\
\hline $\begin{array}{l}\text { \# of } \\
\text { Landmarks }\end{array}$ & 4 & 4 & 3 & 4 & 3 & 3 & 4 \\
\hline TRE (mm) & 3.61 & 2.42 & 3.95 & 3.53 & 3.05 & 2.15 & 1.62 \\
\hline
\end{tabular}


Our registration technique achieves a mean TRE of $2.90 \pm 0.80 \mathrm{~mm}$. These results are comparable to those presented in [6] where the TRE ranges from 2 to $4 \mathrm{~mm}$ for the registration of ultrasound and MRI images of the liver.

\subsection{Visualization of hepatic arteries}

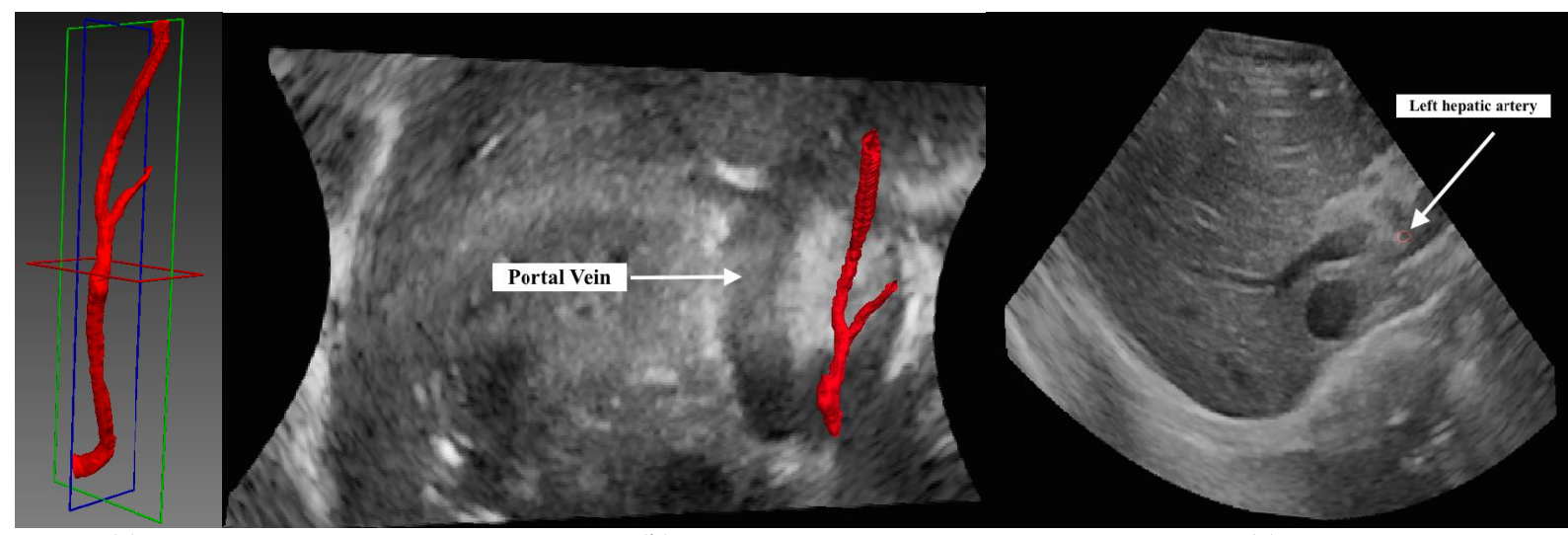

(a)

(b)

(c)

Figure 6- (a) 3D mesh of the pig's hepatic arteries from MRA. (b) Visualization of the 3D arterial mesh within 3D ultrasound, where the hepatic artery accompanies the portal vein. (c) Visualization on an axial slice of the 3D ultrasound. The white arrow points to a mesh cross-section of the left hepatic artery.

Finally, we used the previously computed transformation to integrate the $3 \mathrm{D}$ mesh of the hepatic arteries into the ultrasound volume. Figure 6 depicts the visualization of the hepatic arteries within 3D ultrasound. Figure 6a shows the 3D mesh from MRA, while Figure $\mathbf{6 b}$ and $\mathbf{6 c}$ illustrate the visualization capabilities allowed by our pipeline with live imaging. Figure $\mathbf{6 b}$ shows the 3D mesh on a coronal slice. As expected, the hepatic arteries follow the portal system. On Figure $6 \mathbf{c}$, the white arrow points to the localization of the left hepatic artery on an axial slice of the 3D ultrasound.

\section{CONCLUSION}

We presented an automated pipeline that enables the visualization of hepatic arteries automatically segmented from arterial-phase MRA within a 3D ultrasound after an ultrasound-portal phase MRA registration and an arterial-portal phase alignment for intra-arterial procedures. This approach achieves the combination of the visualization capabilities and vascular information of MRI with ultrasound, which is less expensive and easier to use in an intra-operative context.

Our proposed approach facilitates the acquisition of Doppler measurements by precisely localizing the vessels on live images, especially in patients with anatomical variants. Such a tool would be useful within the context of intra-arterial therapies such as chemoembolizations, where real-time feedback on blood flow velocities can be helpful for the modulation of the injection rate and volume in different segment which in turn are crucial to optimize the treatment of the targeted nodules. Further work will focus on testing and improving the workflow and on improving the performance of the US-MRA registration process.

\section{ACKNOWLEDGMENTS}

This work was supported by the Canadian Research Chairs, the Natural Sciences and Engineering Research Council of Canada and the MEDITIS training program. 


\section{REFERENCES}

[1] Ferlay, J., Soerjomataram, I., Ervik, M., et al. "GLOBOCAN 2012 v1.0, Cancer Incidence and Mortality Worldwide: IARC CancerBase No. 11," Lyon, France: International Agency for Research on Cancer 2013, http://globocan.iarc.fr (August 2015).

[2] Arai, Y., Takeuchi, Y., Inaba, Y., Yamaura, H., Sato, Y., Aramaki, T., Matsueda, K., and Seki, H., "Percutaneous catheter placement for hepatic arterial infusion chemotherapy," Techniques in vascular and interventional radiology 10, 30-37 (2007).

[3] Liapi, E. and Geschwind, J. F., "Chemoembolization for primary and metastatic liver cancer," Cancer journal 16, 156-162 (2010).

[4] Marelli, L., Stigliano, R., Triantos, C., Senzolo, M., Cholongitas, E., Davies, N., Tibballs, J., Meyer, T., Patch, D.W. and Burroughs, A.K., "Transarterial therapy for hepatocellular carcinoma: Which technique is more effective ? A systematic review of cohort and randomized studies," Cardiovascular and interventional radiology 30, 6-25 (2007).

[5] Lange, T.,Eulenstein, S., Hünerbein, M.,Lamecker, H. and Schlag, P.M., "Augmenting Intraoperative 3D Ultrasound with Preoperative Models for Navigation in Liver Surgery." Proc. Medical Image Computing and Computer-Assisted Intervention (MICCAI 2004), 534-541 (2004).

[6] Porter, B.C., Rubens, D.J., Strang, J.G., Smith, J., Totterman, S. and Parker K.J., "Three-Dimensional Registration and Fusion of Ultrasound and MRI Using Major Vessels as Fiducial Markers," IEEE Transactions on Medical Imaging 20(4), 354-359 (2001).

[7] Fuerst, B., Wein, W., Müller, M. and Navab, N., "Automatic ultrasound-MRI registration for neurosurgery using the 2D and 3D LC2 Metric," Medical Image Analysis 18, 1312-1319 (2014).

[8] De Leener, B., Cohen-Adad, J. and Kadoury, S., "Automatic Segmentation of the Spinal Cord and Spinal Canal Coupled With Vertebral Labeling," IEEE Transactions on Medical Imaging 34(8),1705-1718 (2015).

[9] Badoual, A., Gerard, M., De Leener, B., Abi-Jaoudeh, N. and Kadoury, S., "3D Vascular path planning of chemoembolizations using segmented hepatic arteries from MR angiography," Proc. IEEE Intl Symp Biomedical Imaging, (2016).

[10] Fecamp, V., Sotiras, A., and Paragios, P., "Modular linear iconic matching using higher order graphs," Proc. IEEE Intl Symp. Biomedical Imaging (ISBI), 1097-1101 (2015). 